\title{
Gain-Maximizing Dissolved Oxygen Control Using Discrete-Time Adaptive Broken Line Fitting in the Activated Sludge Process ${ }^{\dagger}$
}

\author{
Ryohei TAnuma*, Kosei Sasaki* and Iwao Matsunaga**
}

This paper describes the study of a dissolved oxygen (DO) concentration control in an activated sludge process. The DO concentration at a specific point is controlled around the time-varying set point, which is so manipulated that the gain of the DO concentration with respect to the aeration air flow rate becomes maximum. A new discrete-time algorithm is developed for this control. This algorithm is based on high-pass filters characterized by the parallel configuration of a time lag and a moving average, a difference-form broken line fitting for the gain maximization, and an adaptive PI control, with which the DO concentration is controlled around the time-varying set point. Experiments in a pilot-scale plant show that this control algorithm performs satisfactorily to be applied in actual plants.

Key Words: activated sludge, dissolved oxygen control, gain maximization, adaptive control

\section{Introduction}

The activated sludge process is a biological process which treats industrial and domestic waste water. In this process, although DO control has been developed preliminarily for energy saving, it has been recognized empirically that the DO control can also improve the water quality of the effluent. As is shown in Fig. 1, the DO concentration is almost zero near the inlet of the aeration tank because of high oxygen demand-the anoxic

$\uparrow$ Presented at 6th SICE Symposium on Adaptive Control (1986•1)

* Fuji Electric Corporate Research and Development Ltd., 2-2-1, Nagasaka, Yokosuka, Kanagawa 240-01, Japan

** Fuji Electric Co., Ltd., 12-1, Yurakucho 1-chome, Chiyoda-ku, Tokyo 100, Japan

(Received April 13, 1989)

(Revised December 14, 1989)

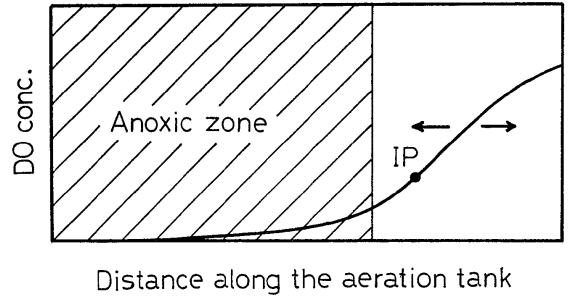

Fig. 1 DO profile in the aeration tank

zone is formed-and increases toward the outlet as reactions proceed. As the aeration air flow rate increases, the DO profile shifts toward the inlet, and the anoxic zone becomes short. Since this process is the mixed culture of aerobic microorganisms, it seems that the shorter the length of the anoxic zone, the better the process conditions. A recent study ${ }^{1)}$, however, shows that the anoxic zone itself suppresses the growth of some filamentous bacteria, which severely lower the quality of effluent. We can hence deduce that the DO control can improve the effluent quality if it maintains an appropriate anoxic zone length.

We have developed a gain-maximizing DO control system, which regulates the anoxic-zone length ${ }^{3)-5)}$. In this system, the DO concentration at a specific point is adjusted in order to maximize the gain of the $\mathrm{DO}$ concentration with respect to the aeration air flow rate. Since the gain becomes maximum just above the minimum DO level to ensure oxic environment, the gain maximization provides the state where the anoxic zone ends just before the control point ${ }^{4)}$. The anoxic-zone length is thereby controlled, and the length is adjustable by selecting the sensor location. Unlike an inflection point (IP) method ${ }^{2)}$, where the IP position of 
the DO profile (Fig. 1) is detected and controlled with multi-point DO measurements, the gain-maximizing method is reliable because it uses only a single DO sensor.

In this paper, we describe the study of this control using a discrete-time algorithm, which is more suitable for computer programming than the continuous-time versions we have reported previously ${ }^{3), 4}$.

\section{Gain Maximization and the DO Profile}

Although the gain-maximizing method does not directly control the IP position, this method is approximately equivalent to the IP method. This can be graphically explained with the CFZ surface in Fig. 2, where $C$ is the $\mathrm{DO}$ concentration, $F$ is the aeration air flow rate, and $Z$ is the distance from the inlet. On this surface, any curve parallel to the $Z C$ plane represents a $D O$ profile; any curve parallel to the $C F$ plane (the $C-F$ curve) represents a static relation between $C$ and $F$. On the same surface, the line MG passes through the maximumgain points (the IPs of the $C-F$ curves); the dotted line MS passes through the IPs of the DO profiles. As can be seen from the figure, $M G$ and $M S$ are close to each other. Consequently, if $C$ at a specific point (e.g. $Z=Z_{m}$ ) is controlled at the value of gain maximization, the IP of the DO profile will be fixed at about the same point.

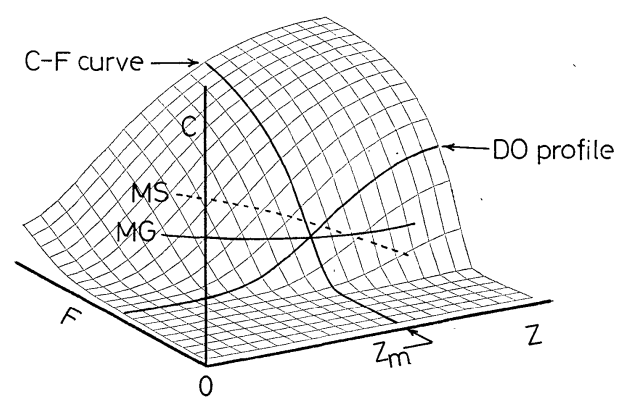

Fig. 2 Perspective of the $\mathrm{CFZ}$ surface

\section{Adaptive Broken Line Fitting}

The $C-F$ curve observed in a pilot-scale plant (to be described later) is shown in Fig. 3. As $F$ increases, the curve changes from concave to convex at the level of the maximum gain (MGL). The

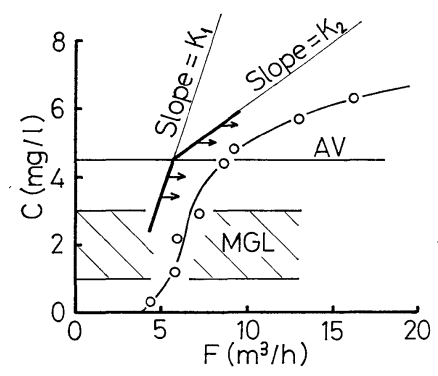

Fig. 3 Observed $C-F$ curve and the broken line

objective of the control is to maintain $C$ around MGL. For the gain maximization, we consider broken-line fitting. As shown in Fig. 3, the broken line to be fitted to the curve consists of two linear parts; let the slopes of the lower and the upper linear parts be $K_{1}$ and $K_{2}$, respectively. Suppose that $C$ fluctuates over an appropriate range, and the breakpoint of the line is placed at the average level (AV) of $C$. If the two slopes can be identified, the gain maximization is achieved through adjusting the DO level so as to equalize the two slopes.

In order to derive the algorithm for the broken line fitting, we start with a linear model. It is known that the response of $C$ to $F$ is described by a first-order linear model ${ }^{6}$, which can be expressed in the form of the difference equation ${ }^{5)}$ :

$$
Q \Delta y(k)+y(k)=K u(k),
$$

where $y(k)$ is the difference between $C$ and its average, $u(k)$ is the difference between $F$ and its average, $\Delta y(k)=y(k+1)-y(k), K$ is the gain, and $Q$ is the coefficient related to the time constant and the sampling time. The procedure of obtaining $u(k)$ and $y(k)$ from $F$ and $C$, respectively, is a kind of high-pass filtering. This filtering is very important in this system and its detail will be described later. Because $K$ appears explicitly in the difference form, this equation is more suitable to be extended to the algorithm for the gain maximization than a conventional shift equation ${ }^{5}$.

The parameters $Q$ and $K$, which are time varying because of nonlinearity, can be regarded as functions of $C$. If we assume that the parameters take two distinct values, depending on the DO level, the switching parameter model is obtained:

$$
Q_{i} \Delta y(k)+y(k)=K_{i} u(k)
$$




$$
i=\left\{\begin{array}{l}
1 \text { for } s(k) \leqq 0 \\
2 \text { for } s(k)>0,
\end{array}\right.
$$

where $s(k)=y(k+1)+y(k)$.

This equation is identical to the following expression :

$$
\boldsymbol{p} \boldsymbol{\phi}(k-1)+y(k-1)=0
$$

where

$$
\begin{aligned}
& \boldsymbol{p}=\left[\begin{array}{llll}
Q_{1} & Q_{2} & K_{1} & K_{2}
\end{array}\right] \\
& \phi(k-1)=\left\{\begin{array}{l}
{\left[\begin{array}{l}
\Delta y(k-1) 0-u(k-1) 0 \\
\quad \text { for } s(k-1) \leqq 0
\end{array}\right.} \\
{\left[\begin{array}{l}
0 \Delta y(k-1) 0-u(k-1) \\
0
\end{array}\right]^{\mathrm{T}}} \\
\text { for } s(k-1)>0 .
\end{array}\right.
\end{aligned}
$$

The equation error $e$ is defined as

$$
e=\boldsymbol{p}_{e}(k) \boldsymbol{\phi}(k-1)+y(k-1),
$$

where $\boldsymbol{p}_{e}(k)$ is the adjustable parameter vector,

$$
\boldsymbol{p}_{e}(k)=\left[Q_{e 1}(k) Q_{e 2}(k) K_{e 1}(k) K_{e 2}(k)\right] \text {. }
$$

Subtracting Eq. (3) from Eq. (4), we obtain the error equation,

$$
e=\left[\boldsymbol{p}_{e}(k)-\boldsymbol{p}\right] \boldsymbol{\phi}(k-1) .
$$

The parameters can hence be identified by the recursive algorithm,

$$
\begin{aligned}
& \boldsymbol{e}_{0}=\boldsymbol{p}_{e}(k-1) \boldsymbol{\phi}(k-1)+y(k-1) \\
& \boldsymbol{p}_{e}(k)=\boldsymbol{p}_{e}(k-1)-\frac{\boldsymbol{G} \boldsymbol{\phi}(k-1) e_{0}}{1+\boldsymbol{\phi}^{T}(k-1) \boldsymbol{G} \boldsymbol{\phi}(k-1)},
\end{aligned}
$$

where $e_{0}$ is the a-priori value of $e$, and $\boldsymbol{G}$ is the adaptation gain matrix. ${ }^{7)}$ Since $K_{e 1}(k)$ and $K_{e 2}(k)$, the last two members of the vector $\boldsymbol{p}_{e}(k)$, correspond to the slopes of the lower and upper linear parts, respectively of the broken line, they can be used for the gain maximization. Hereafter, we refer to the above identification algorithm as the adaptive broken line fitting (ABLF).

In the $A B L F$, various disturbances which the plant receives act almost equally on $K_{e 1}$ and $K_{e 2}$, causing common errors in the two gains. The common errors can be cancelled by subtracting the two gains for the gain maximization. This is the main reason why the ABLF can ensure reliable gain-maximizing control.

\section{The Control System}

\subsection{Concept}

The gain-maximizing control system, shown in Fig. 4, consists of the DO-control loop and the gain-maximizing loop. The former controls $C$ around the adjustable set point $C_{r}$, and the latter manipulates $C_{r}$ to maximize the gain. The input $F$ and the output $C$ are converted into the zero-mean signals, $u$ and $y$ respectively, by the high-pass filters. On receiving $u$ and $y$, the ABLF determines $Q_{e 1}, Q_{e 2}, K_{e 1}$, and $K_{e 2}$. The controlled variable in the gain-maximizing loop is $c_{v}$ defined as

$$
c_{v}=\left[K_{e 2}(k)-K_{e 1}(k)\right] / K_{e}(k)
$$

where

$$
K_{e}(k)=\left[K_{e 1}(k)+K_{e 2}(k)\right] / 2 .
$$

Since $c_{v}=0$ is approximately identical to $\left(\mathrm{d}^{2} C /\right.$

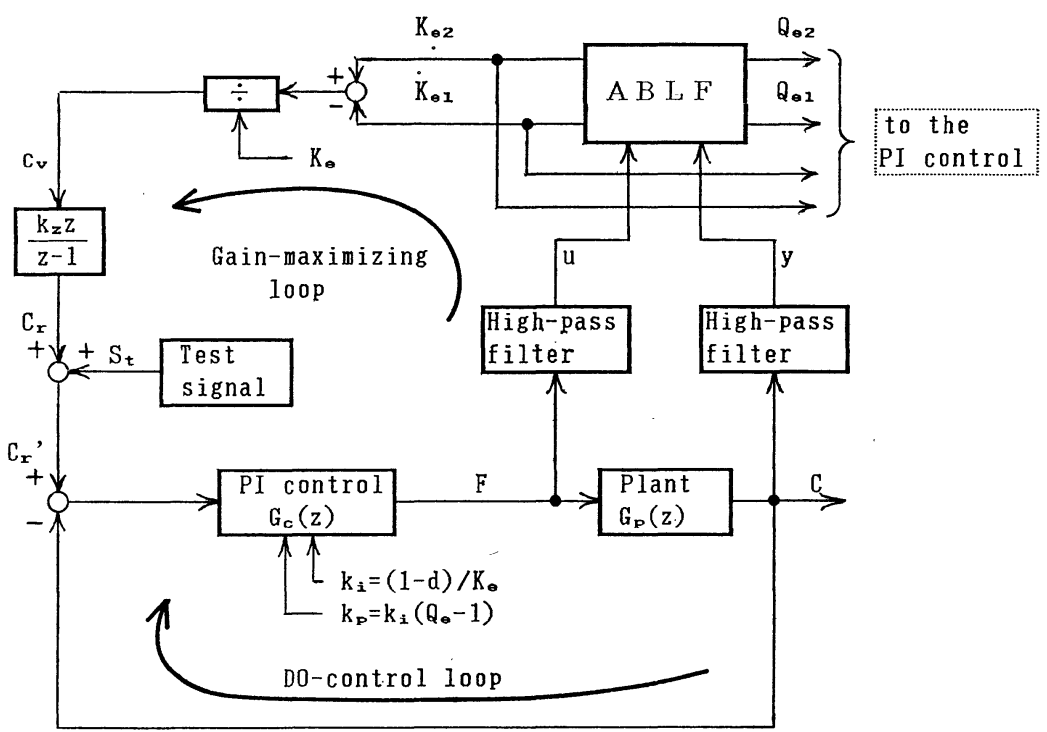

Fig. 4 Gain-maximizing DO control system 
$\left.\mathrm{d} F^{2}\right)=0$, controlling $c_{v}$ at zero with manipulating $C_{\tau}$ results in the gain maximization. For this control, the integral action ( $k_{z}$ is its gain.) is used because $c_{v}$ is often quite noisy. For $C$ to fluctuate over an appropriate range, the test signal $S_{t}$ is applied to $C_{r}$. The $\mathrm{DO}$ concentration $C$ tracks the perturbed set point $C_{r}^{\prime}=C_{r}+S_{t}$ with an adaptive PI control.

In this system, $S_{t}$ is the zero-mean random value sequence shown in Fig. 5. The value of $S_{t}$ is updated at the intervals of $\tau_{t}$; the periods of $S_{t}$ are thereby limited to $2 \tau_{t}$ and its integral divisors. The amplitude of $S_{t}$ is specified in terms of its standard deviation $D_{s}$.

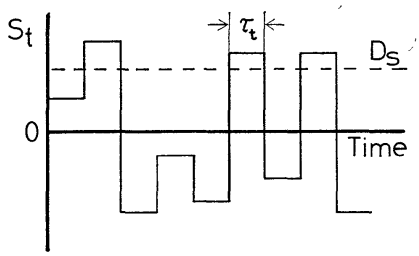

Fig. 5 Zero-mean random value sequence applied to $C_{r}$ as the test signal $S_{t}$.

We usually take the same sampling time for the DO-control loop and the gain-maximizing loop. Although there is no clear criterion to determine the sampling time, it should be taken into account that data must be sampled at least 3-4 times within $\tau_{t}$, whereas too short sampling time makes the difference $\Delta y$ noisy.

In order to keep the gain-maximizing control in high performance, the following two conditions must be satisfied: $C$ fluctuates over a constant range (Cond. A); the fluctuating range of $C$ and $F$ are divided equally into the lower and upper region (Cond. B).

\subsection{Adaptive PI control}

Satisfying Cond. A requires the dynamics of the DO control loop to be constant. The transfer function of the process on the basis of the difference Eq. ( 1 ) is

$$
G_{p}(z)=\frac{K z^{-1}}{1+(Q-1)\left(1-z^{-1}\right)} .
$$

The transfer function of the PI algorithm used here is

$$
G_{c}(z)=\frac{1+\left(k_{p} / k_{i}\right)\left(1-z^{-1}\right)}{\left(1 / k_{i}\right)\left(1-z^{-1}\right)},
$$

where $k_{p}$ is the proportional gain, and $k_{i}$ is the integral gain. Now we assume that the parameter identification is completed; that is, $Q=Q_{e}=\left(Q_{e 1}+\right.$ $\left.Q_{e 2}\right) / 2$, and $K=K_{e}=\left(K_{e 1}+K_{e 2}\right) / 2$. If we determine $k_{p}$ as

$$
k_{e}=\left(Q_{e}-1\right) k_{i},
$$

the open loop transfer function, $G_{c}(z) G_{p}(z)$, is simplified to

$$
G_{c}(z) G_{p}(z)=\frac{K k_{i} z^{-1}}{1-z^{-1}}
$$

and the closed loop transfer function $G_{L}(z)$ then becomes

$$
\begin{aligned}
G_{L}(z) & =\frac{G_{c}(z) G_{p}(z)}{1+G_{c}(z) G_{p}(z)} \\
& =\frac{K k_{i} z^{-1}}{1-\left(1-K k_{i}\right) z^{-1}} .
\end{aligned}
$$

In addition, if we determine $k_{i}$ as

$$
k_{i}=(1-d) / K_{e},
$$

the dynamics of the DO control loop are fixed:

$$
G_{L}(z)=\frac{1-d}{z-d},
$$

where $d$ is the desired pole value of the closed loop. Hence, under the adaptive PI control in which $k_{p}$ and $k_{i}$ are determined by Eqs. (11) and (14), we can specify the width of the output variationcan by adjusting the values of $\tau_{t}, D_{s}$, and $d$.

\subsection{High-pass filtering for ABLF}

The control system undergoes not only the test signals, but also various disturbances such as load variation, temperature variation, and the gain-maximizing action itself. Since the period of the test signal is usually far shorter than those of the disturbances, the variations of $F$ and $C$ are classified into the high-frequency (HF) test-signal components and the low-frequency (LF) disturbance components. In this system, it is very important to filter out the LF components through obtaining $u$ and $y$ from $F$ and $C$, respectively. If the filtering is insufficient, $u$ and $y$ will often be biased, and Cond. B cannot be satisfied.

To ensure this high-pass filtering, we have used the following algorithm:

$$
\begin{aligned}
\zeta(k)= & \eta(k-m / 2) \\
& -\frac{\eta(k)+\eta(k-1)+\cdots+\eta(k-m+1)}{m}
\end{aligned}
$$

where the pair of $\zeta$ and $\eta$ is that of $y$ and $C$, or 


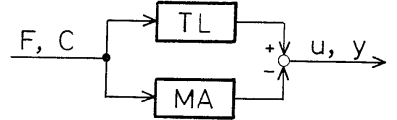

Fig. 6 Filtering algorithm expressed as the parallel configuration of a time lag (TL) and a moving average (MA)

$u$ and $F$, respectively, and $m$ is the number of the data, which is taken as an even number. This filtering algorithm is expressed by the parallel configuration of the time lag (TL) and the moving average (MA) as shown in Fig. 6. The transfer functions of TL and MA, $G_{T L}(z)$ and $G_{M A}(z)$ respectively, are

$$
\begin{aligned}
& G_{T L}(z)=z^{-m / 2}, \\
& G_{M A}(z)=\frac{1-z^{-m}}{m\left(1-z^{-1}\right)} .
\end{aligned}
$$

The frequency response of MA is expressed by

$$
\begin{aligned}
G_{M A}\left(\mathrm{e}^{j \omega T}\right) & =\frac{1-\mathrm{e}^{-j m \omega T}}{m\left(1-\mathrm{e}^{-j \omega T}\right)} \\
& =\frac{1-\mathrm{e}^{-j m \omega T}}{m\left[j \omega T+(\omega T)^{2} / 2 !+\cdots\right]},
\end{aligned}
$$

where $\omega$ is the angular frequency, and $T$ is the sampling time. Since the value of $T$ is so determined that $T \ll \tau=2 \pi / \omega$ holds, $\omega T=2 \pi(T / \tau) \ll 1$, and then,

$$
\begin{aligned}
G_{M A}\left(\mathrm{e}^{j \omega L}\right) & \fallingdotseq \frac{1-\mathrm{e}^{-j \omega L}}{j \omega L} \\
& =[\sin \omega L-j(1-\cos \omega L)],
\end{aligned}
$$

where $L=m T$. We determine the value of $L$ as $L=n \tau_{H F}=2 \pi n / \omega_{H F}(n=1,2, \cdots)$, where $\tau_{H F}$ and $\omega_{H F}$ are the periods and the angular frequencies, respectively of the $\mathrm{HF}$ components; this is possible because the values of $\tau_{H F}$ can be specified by $S_{t}$. If $L$ is thereby determined, $\omega_{H F} L=2 \pi L / \tau_{H F}=2 n \pi$ $(n=1,2, \cdots)$; that is,

$$
G_{M A}\left(\mathrm{e}^{j \omega H F}\right)=0 \text {. }
$$

This means that the HF components are rejected almost completely by MA and appear only in the output of TL. Consequently, the HF components pass the filter with the time lag of $L / 2$.

Now let us consider the LF components. From Eq. (20) we obtain

$$
\begin{aligned}
G_{M A}\left(\mathrm{e}^{j \omega T}\right) & \fallingdotseq \frac{2}{\omega L} \sin \left(\frac{\omega L}{2}\right) \cdot\left[\cos \left(\frac{\omega L}{2}\right)-j \sin \left(\frac{\omega L}{2}\right)\right] \\
& =\frac{2}{\omega L} \sin \left(\frac{\omega L}{2}\right) \cdot \mathrm{e}^{-j \omega(L / 2)} .
\end{aligned}
$$

Equation (21) can be rewritten as

$$
G_{M A}\left(\mathrm{e}^{j \omega T}\right) \fallingdotseq\left[1-\frac{1}{3 !}\left(\frac{\pi L}{\tau}\right)^{2}+\frac{1}{5 !}\left(\frac{\pi L}{\tau}\right)^{4}-\cdots\right] \cdot \mathrm{e}^{-j \omega(L / 2)}
$$

The value of $L$ is typically taken as $2 \mathrm{~h}$. In the activated sludge process, the period of main load variation is 12 or $24 \mathrm{~h}$. If we suppose the former as the smallest period of the LF components,

$$
(1 / 3 !)\left(\pi L / \tau_{L F}\right)^{2} \leqq 0.046 \ll 1,
$$

where $\tau_{L F}$ are the periods of the LF components. Hence,

$$
\begin{aligned}
G_{M A}\left(\mathrm{e}^{j \omega} L F T\right. & \fallingdotseq \mathrm{e}^{-j \omega L F}(L / 2) \\
& =G_{T L}\left(\mathrm{e}^{j \omega L F T}\right),
\end{aligned}
$$

that is, the LF components appear commonly in the output of TL and MA. Consequently, the LF components can be filtered out almost completely with the high-pass filters.

\section{Experimental}

The pilot-scale activated sludge plant we have used is shown schematically in Fig. 7. This plant treats about $10 \mathrm{~m}^{3} /$ day of waste water from the cafeteria of our laboratory. Activated sludge removes pollutant in the aeration tank and is then separated from purified water in the sedimentation tank. The settled sludge returns to the aeration tank. The aeration tank is divided into four compartments by partitions to prevent complete mixing.

The DO meter measures the DO concentration in the third compartment from the inlet. Data acquisition and control calculations are performed with the $\mathrm{AD} / \mathrm{DA}$ converter and the micro-computer, linked by GP-IB bus. The DO-concentration data acquired every $1-2 \mathrm{~s}$ are first averaged for one minute. Averaged DO data are thereby obtained every one minute, and some of them are sampled (typically every five minutes) for the control calculations. This averaging can reduce the noise level of DO data while preserving the process dynamics because the averaging time is far shorter than the time constant of the process.

The outputs of the control calculations are given to the air flow rate control loop as its desired values. This control loop consists of the orifice flow meter, digital one-loop controller, inverter, 


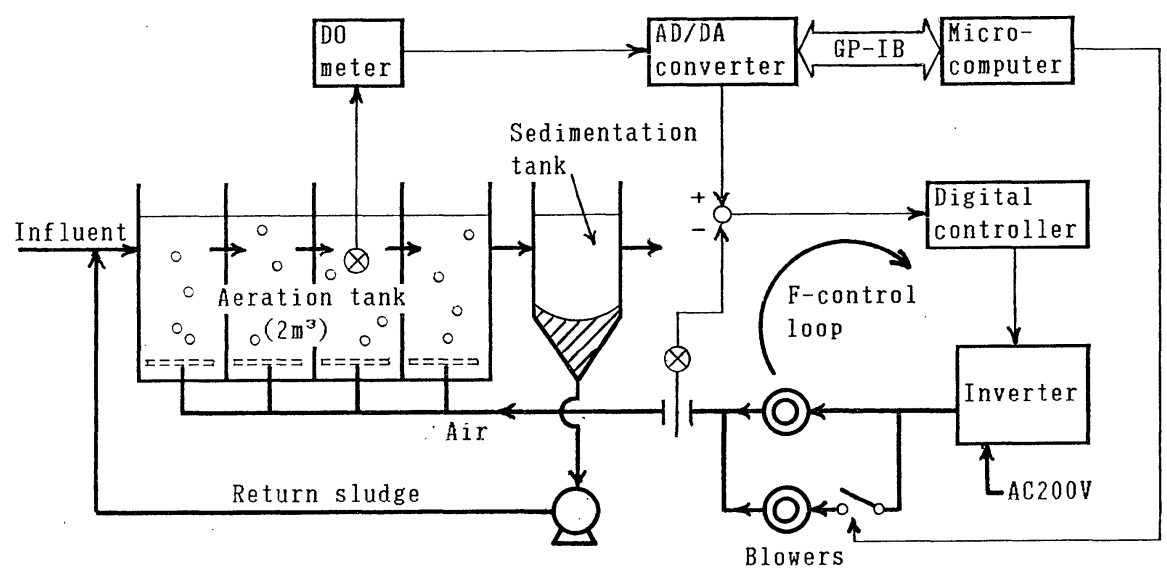

Fig. 7 Experimental plant

and two blowers, one of which is switched on and off depending on the air flow rate.

\section{Control Results and Discussion}

We performed two control experiments, Control $I$ and $I I$, whose results are shown in Fig. 8 and 9 respectively. In these experiment, $T=5 \mathrm{~min}$, and $m=24 ; L=m T=120 \mathrm{~min}$. The test signal was specified as follows: $\tau_{t}=30 \mathrm{~min}$ and $D_{s}=1 \mathrm{mg} / 1$. The gain matrix $\boldsymbol{G}$ was the diagonal with the diagonal elements $g_{11}=g_{22}=300$ and $g_{33}=g_{44}=100$. These values are based on the dimensionless variables $c=C(\mathrm{mg} / \mathrm{l}) / 10(\mathrm{mg} / \mathrm{l})$ and $f=F\left(\mathrm{~m}^{3} / \mathrm{h}\right) / 25\left(\mathrm{~m}^{3} / \mathrm{h}\right)$. The value of the integral gain $k_{z}$, which is also based on $c$, was $k_{z}=0.0017$ in Control I, and dou- bled to be $k_{z}=0.0034$ in Control II. The desired pole value was $d=0.78$.

Control I started with $C_{r}$ as low as $0.5 \mathrm{mg} / 1$ and continued for about $300 \mathrm{~h}$. The set point $C_{r}$ reached the values of $1.0-1.5 \mathrm{mg} / 1$ in about $20 \mathrm{~h}$ and became stable there. The settled $C_{r}$ value is in good agreement with MGL in Fig. 3. The values of $K_{e 1}$ and $K_{e 2}$, which were different when $C_{r}$ was low, approached each other as $C_{r}$ reached the settled value.

Although the main reaction in the activated sludge process is the oxidation of organic pollutant by heterotrophs, the oxidation of ammonium ion (nitrification) by autotrophs often occurs concurrently. When Control I was performed, almost
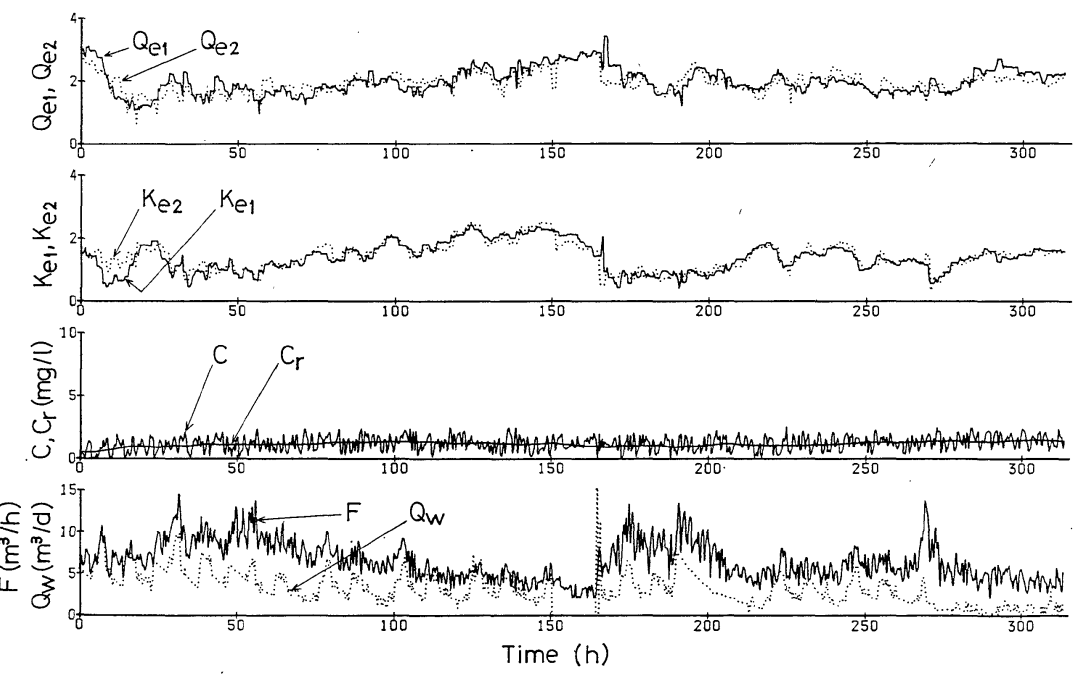

Fig. 8 Control I: no nitrification 

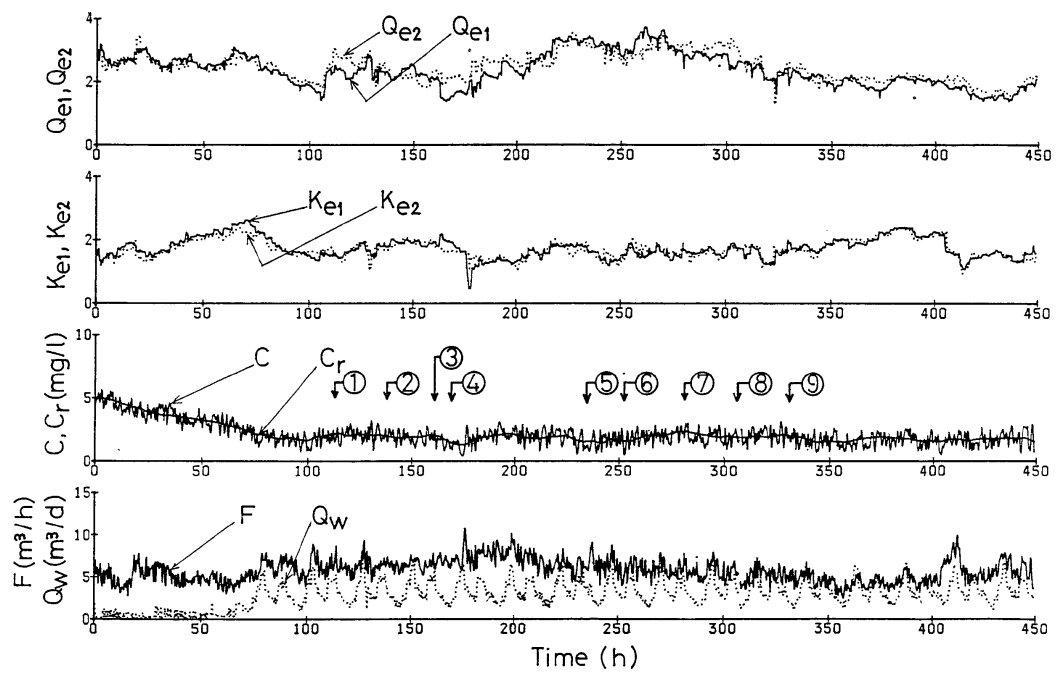

Fig. 9 Control II: the settled $C_{r}$ value is higher than that in Control I because of the nitrification

no nitrification occurred. After that, process conditions were changed to favor nitrification.

We started Conttrol II after making sure that nitrification had occurred. The initial value of $C_{r}$ was $5 \mathrm{mg} / 1$, and the control continued for about $450 \mathrm{~h}$. The set point $C_{r}$ decreased and reached the values of $1.4-2.4 \mathrm{mg} / 1$ in about $100 \mathrm{~h}$. Since the value of $k_{z}$ was doubled, $C_{r}$ fluctuated more. Although the level where $C_{r}$ settled is also in good agreement with MGL in Control II, it is a little higher than the settled value in Control I. This can be explained by the fact that the minimum DO level for oxic environment rises as nitrification autotrophs grow $^{8)}$.

The DO profiles observed in Control II are shown in Fig. 10. The control point is No. 3 compartment. The profile numbers in this figure correspond to the measurement-time numbers from Fig. 9. The bold solid line $\mathrm{DP}_{0}$ shows the average

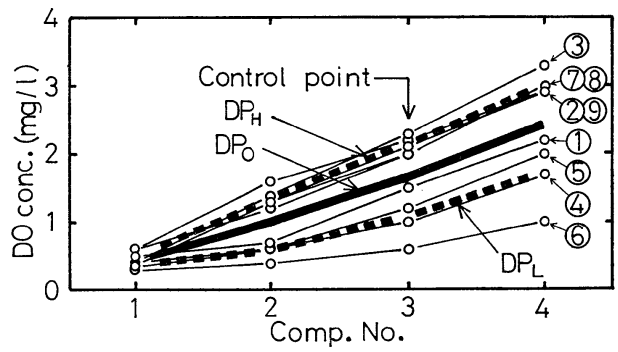

Fig. 10 DO profiles observed in Control II
DO profile. Perturbed by the test signal, the DO profiles are distributed around $\mathrm{DP}_{0}$. The $\mathrm{DO}$ value distribution in No. 3 compartment corresponds to the $C$ variation in Fig. 9. We consider that this kind of short-period DO variation is trivial in the activated sludge processes; that is, the average profile is significant.

Now let us divide the profiles in upper and lower groups separated by $\mathrm{DP}_{0}$. Averaging the profiles in each group, we obtain the dashed lines $\mathrm{DPH}_{\mathrm{H}}$ and $\mathrm{DP}_{\mathrm{L}}$ shown in Fig. 10. We can see that $\mathrm{DP}_{0}$ and $\mathrm{DP}_{\mathrm{L}}$ are a little concave, but $\mathrm{DPH}$ is linear. This suggests that the IPs are fixed between the control point and the outlet.

The DO values in No. 1 and 2 compartments are lower than the control point DO value, which is the minimum DO value for oxic environment. This indicates that anoxic conditions are ensured in No. 1 and 2 compartments.

The profile IPs are not necessarily clear in Fig. 10 because of large backward mixing through the partitions. Similar situations are often encounterd in actual plants. Even in such a case, this method can successfully control the anoxic zone length as described above.

The gain-maximizing DO control is very useful for nitrification processes. Since the steep rise in DO concentration will be observed at a nitrification end point, the reaction is controllable with 
this method. In fact, the nitrification was successfully controlled over the period of Control III. For further details, refer to our previous presentation ${ }^{5}$.

In these experiments, the influent flow rate $Q_{W}$ varied greatly (such a flow pattern is typical in actual plants). Though the values of the parameters depend on $Q_{W}$, the gain-maximizing action was independent of the load variation and quite stable. We consider that the stability is not only due to the common-error cancelling, but also the high-pass filtering as well as the adaptive PI control.

\section{Conclusions}

In the experiments of the new gain-maximizing DO control system, the gain maximization was successfully performed under great load variation, and the controls were stable for a long period of time. The observed DO profiles show that the IP position and the anoxic zone length were controlled. From these results, we conclude that the new system performs satisfactorily to be applied in actual plants.

\section{References}

1) J. Wanner, J. Chudoba, K. Kucman and L. Proske: Control of Activated Sludge Filamentous Bulking
-VII. Effect of Anoxic Conditions, Water Research, 21-12, 1447/1451 (1987)

2) G. Olsson and J.F. Andrews: The Dissolved Oxygen Profile-a Valuable Tool for Control of the Activated Sludge Process, Water Research, 12-11, 985/1004 (1978)

3) R. Tanuma, K. Sasaki and I. Matsunaga: GainMaximizing Dissolved Oxygen Control Using Adaptive Broken Line Fitting in the Activated Sludge Process, Proceedings of 5th SICE Symposium on Adaptive Control, 17/22 (1985)

4) R. Tanuma, K. Sasaki and I. Matsunaga: GainMaximizing Dissolved Oxygen Control in the Activated Sludge Process, Instrument and Control of Water and Wastewater Treatment and Transport System, 261/268, Pergamon Press (1985)

5) R. Tanuma, K. Sasaki and I. Matsunaga: GainMaximizing Dissolved Oxygen Control Using Discrete-Time Adaptive Broken Line Fitting in the Activated Sludge Process, Proceedings of 6 th $\mathrm{SICE}$ Symposium on Adaptive Control, 65/68 (1986)

6) G. Olsson, L. Rundqwist, L. Eriksson and L. Hall: Self-tuning Control of the Dissolved Oxygen Concentration in Activated Sludge Systems, Instrument and Control of Water and Wastewater Treatment and Transport System, 473/480, Pergamon Press (1985)

7) I. D. Landau and M. Tomizuka: Theory and Practice of Adaptive Control Systems (in Japanese), Ohmu-sha (1981)

8) R. Tanuma, K. Sasaki and I. Matsunaga: GainMaximizing Dissolved Oxygen Control in a $\mathrm{Bi}$ ological Nitrification Process, Proceedings of 20th Annual Conference of Japan Society on Water Pollution Research, 269/270 (1986) 